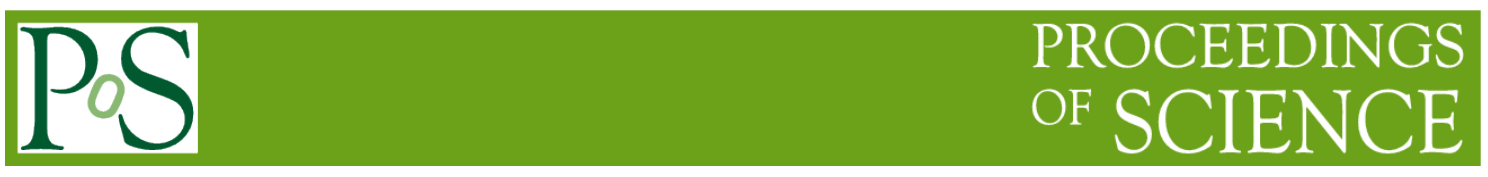

\title{
Passive Detection of Sea Targets by Using Loran-C
}

\author{
Jianing Shi ${ }^{1}$ \\ School of Electronic and Information Engineering, Beihang University \\ No.37, XueYuan Road, HaiDian District, Beijing, 100191, China \\ E-mail: sheboy28@yahoo.com

\section{Wenguang Wang ${ }^{2}$} \\ School of Electronic and Information Engineering, Beihang University \\ No.37, XueYuan Road, HaiDian District, Beijing, 100191, China \\ E-mail: wwenguang@buaa.edu.cn
}

The paper presents a passive detection method in which Loran-C signal is chosen as the noncooperative detection signal to detect sea targets. This method processes the echoes of the Loran-C signals from different stations to implement detection of large sea targets beyond long range. The Loran-C signal and any advantages it has with respect to the passive detection for sea targets are investigated in detail. The paper describes fully the signal processing scheme and the associated techniques. Moreover, simulations are presented in this paper to demonstrate that the method is indeed viable.

ISCC 2015

18-19, December, 2015

Guangzhou, China

\footnotetext{
${ }^{1}$ Speaker

${ }^{2}$ This study is supported by the Pre-research Foundation ( Grand No.9140A07040714HK0109)
} 


\section{Introduction}

Passive coherent location (PCL) systems which are based on third-party electromagnetic signals detect and track target by using time delay and the Doppler shift of the echoes. PCL systems have a lot of advantages, such as anti-jamming, low cost, anti-stealth and so on.

A number of exemplar PCL systems have been proposed in recent years. Howland presents a bistatic radar system using non-cooperative Frequency Modulation (FM) radio signals. The system employs an efficient adaptive filter algorithm to suppress interference and strong clutter signals in the echoes, so that the target could be detected by utilizing Dopplersensitive cross-correlation techniques and Cell Averaging Constant False Alarm Rate (CACFAR) algorithm [1]. The factors that could have impact on the performance of PCL systems, such as waveform properties and geometrical factors, are examined in detail by Griffiths [2-3]. Griffiths compares the sensitivity and coverage performance of PCL systems using different signals including FM radio, cellphone base station and digital audio broadcast (DAB), then analyzes how the waveform and geometrical factors influence the performance of PCL systems. The Lockheed Martin's 'Silent Sentry' system which is designed for air and space surveillance has been widely used. Tan proposes a PCL system using Global System for Mobile communication (GSM) [4], and presents an airborne passive radar using the composite spacetime snapshot signal [5].

The proposed systems always need the Doppler information to implement the detection or tracking, and the radio signals such as FM radio, which have been used for passive detection, always have high carrier frequency. Sea targets which are always in slow motion have small Doppler shifts and the signals with high frequency suffer great influence of sea clutter. Therefore, the proposed systems are not suitable for the detection of sea targets. One existing radio signal suitable for passive detection of sea targets is Loran-C signal. This signal has rich transmitter and it is a valuable resource for passive detection with at least three distinct advantages. First, as a kind of long-range radio navigation signal with low carrier frequency and high transmitting power, Loran-C signal could be transmitted over a long distance accompanied by small multipath interference. Second, multiple coastal stations could be utilized for improving the performance and capability. Third, as the wide application in navigation and fixed signal format, many mature techniques are available for the processing of Loran-C signal. To sum up, Loran-C signal is more suitable for the passive detection of sea targets. This paper describes fully the signal processing scheme and associated techniques.

\section{Loran-c Signal}

The pulse of Loran-C signal could be expressed as [6-7] :

$$
x_{0}(t)=\left\{\begin{array}{cc}
0 & t<\tau \\
A(t-\tau)^{2} \exp \left(\frac{-2(t-\tau)}{65}\right) \sin (0.2 \pi t+p c) & t \geqslant \tau
\end{array}\right.
$$

Where $A$ is the normalized peak amplitude, and $p c$ is the phase coded parameter. The unit of parameter $t$, which is the time, and the parameter $\tau$ which is the envelope-to-cycle difference (ECD), are both us. A group of stations which work together in the Loran-C system is called a station chain. A station chain generally consists of a main station and two deputy stations which transmit the Loran-C signal in different formats and different time. First, users can obtain the arrival time interval between the signals from a main station and those from a deputy station, 
then convert it into a distance difference. Second, the hyperbolic trajectories of the receiver could be obtained based on the distance difference and the transmitters' positions.

Compared with other signals for passive detection, Loran-C signal has lower carrier frequency and a wavelength as long as $3 \mathrm{~km}$; therefore, there is a low attenuation caused by ground absorption when the Loran-C signal transmits. For small targets, the backscatter of Loran-C signal is weak due to the diffraction. But for large targets, such as large cargo ships at sea, the backscatter of Loran-C signal could be strong enough to be detected. As a long-wave signal, Loran-C signal suffered little influence of sea clutter, therefore, it can be used for overthe-horizon passive detection of large targets at sea.

\section{Passive Detection Method}

The received signal which the receiver captures from one of the stations, consists of the direct path signal, the target echoes which reach behind the direct path signal, and other components. The distance difference could be obtained by using the signal processing. As shown in Fig.1(a), the ellipse on which the target locates could be calculated based on the distance difference and the position coordinates of the receiver and transmitter station. After processing the received signals of three stations in the same station chain, a group of elliptic equations about the target position could be obtained, and the target position could be calculated by solving the least square solution, then the target could be detected and located finally, as shown in Fig.1(b).
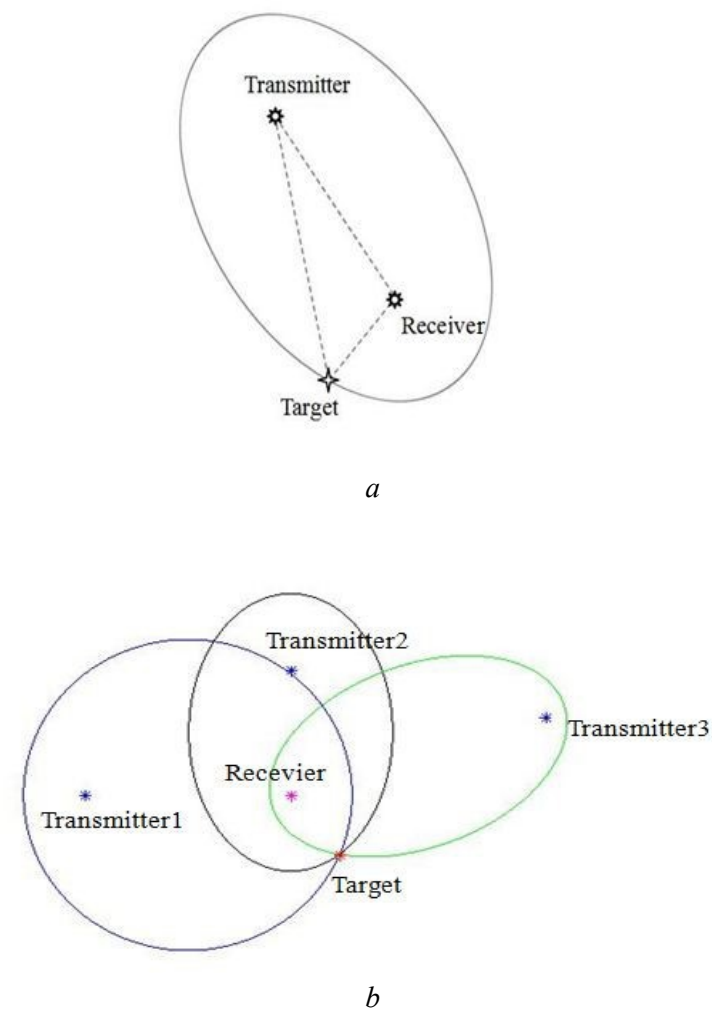

Figure 1: Schematic Diagram of Passive Detection Method

a Geometrical relationship of the target, receiver and single transmitter

b Geometrical relationship of the target, receiver and three transmitters. 


\section{Signalprocessing For Passive Detection}

The received Loran-C signal consists of direct path signals from different transmitters, even from different station chains, target echoes, skywave signal, and noise. The direct path signals from the nearest chain are the strongest component and usually several tens of $\mathrm{db}$ stronger than the target echoes. But the direct path signals from other chains are usually as weak as noise. The skywave signal, which is effected by geography factors and weather conditions, is much stronger than the target echoes. The arrival time of the skywave signal is usually $30 \sim 60$ us later than that of the direct path signals; therefore, the direct path signals from the nearest chain and the skywave signal are the main interference to the target detection.

The interference should be suppressed or removed in order to extract the target echoes. The signal processing scheme of Loran-C signal for target detection is shown in Fig. 2. First, the signals from different stations are separated according to prior knowledge. Then, the target detection and location are accomplished after interference suppression, correlation and peak detection.



Figure 2: Signal Processing Scheme

The target echoes are much weaker than the direct path signals and the skywave signals, therefore the interference suppression is the main challenge of the signal processing. Mohammed proposes multiple delay estimation methods for Loran-C signals, including Auto Regressive Moving Average (ARMA), Auto Regressive (AR), Inverse Fast Fourier Transform (IFFT) and other methods [6]. The received signals from one station could be represented in the time domain by

$$
x_{c}(t)=A_{g} x_{0}\left(t-\tau_{g}\right)+A_{s} x_{0}\left(t-\tau_{s}\right)+\sum_{i=1}^{N} A_{t i} x_{0}\left(t-\tau_{i}\right)+n(t)
$$

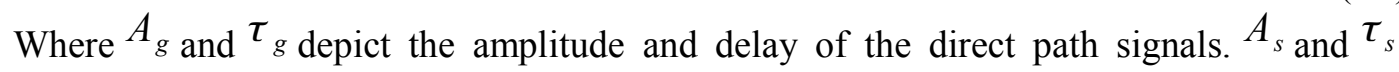
depict the amplitude and delay of the skywave. $A_{t i}$ and $\tau_{i}$ depict the amplitude and delay of the target echo. $n(t)$ is the total noise and other interference. The equivalent representation of the received signal in the frequency domain is given by

$$
\begin{gathered}
X_{c}(f)=A_{g} X_{0}(f) \exp \left(j 2 \pi f \tau_{g}\right)+A_{s} X_{0}(f) \exp \left(j 2 \pi f \tau_{s}\right) \\
+\sum_{i=1}^{N} A_{t i} X_{0}(f) \exp \left(j 2 \pi f \tau_{i}\right)+N(f)
\end{gathered}
$$

Where $X_{0}(f)$ represents the Fourier transform of the normalized standard Loran-C signal which has narrow band, and $N(f)$ which represents the transform of $n(t)$ can be effectively removed by employing a windowing function in the frequency domain. A Hanning window of $50 \mathrm{kHz}$ bandwidth which gives the best performance after numerous computer simulations is selected in this paper. When $X_{c}(f)$ is divided by $X_{0}(f)$, the amplitudes and delays of the direct path signal and the skywave signal are then obtained by taking the inverse fast Fourier transform (IFFT). That is given by 


$$
F^{-1}\left[\frac{X_{c}(f)}{X_{0}(f)}\right]=A_{g} \delta\left(t-\tau_{g}\right)+A_{s} \delta\left(t-\tau_{s}\right)+\sum_{i=1}^{N} A_{t i} \delta\left(t-\tau_{i}\right)
$$

A lot of preliminary experimental results lead to the conclusion that the IFFT method is effective to estimate the parameters of the direct path signal and the skywave signal, it can be chosen to generate the synchronized direct path signals. Gradient Adaptive Lattice (GAL) filter then is used for adaptive filtering in order to separate strong interference and the target echoes. Both literature and simulation results show that GAL adaptive filtering can effectively separate the strong interference and the weak target echoes [1]. The filter algorithm is described by Haykin and is not repeated here [8]. The processing scheme of strong interference suppression used in passive target detection is shown in Fig. 3.

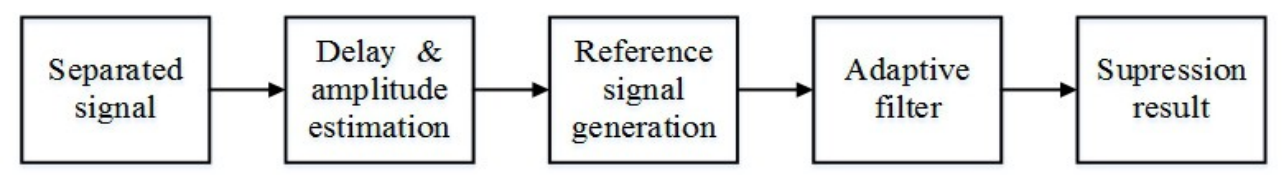

Figure 3: Strong Interference Suppression Scheme

After strong interference suppression for each station's signal, the correlation and peak detection can be used to obtain the arrival time interval between the direct path signals and the target echoes. According to the time delay and the stations' coordinates, it is possible to establish elliptic equations about the target position, and the target could be detected and located by solving elliptic equations based on the least squares solution.

\section{Simulation Result}

The capability of the proposed method is validated through simulation. The simulation environment which is shown in Fig. 4, consists of one target, three stations and one receiver. The position parameters of the simulation, according to typical geometrical configuration of Loran-C system, are given in Table 1. Received signals from different stations are modeled based on the standard Loran-C signal. The simulated data and processing results of the main station are shown in Fig. 5. The results demonstrate that the target echoes are easier to be detected after the effective suppression of the strong interference. According to the processing results of the signals from different stations, the target's position is calculated as $(200.52 \mathrm{~km}$, $-201.99 \mathrm{~km}$ ), which affirms that the method has the capability for detection and location for sea targets.

\begin{tabular}{|c|c|}
\hline Name & Position \\
\hline Main Station & $(0 \mathrm{~km}, 210 \mathrm{~km})$ \\
\hline First Deputy Station & $(520 \mathrm{~km}, 130 \mathrm{~km})$ \\
\hline Second Deputy Station & $(-420 \mathrm{~km}, 100 \mathrm{~km})$ \\
\hline Receiver & $(0 \mathrm{~km}, 0 \mathrm{~km})$ \\
\hline Target & $(200 \mathrm{~km},-200 \mathrm{~km})$ \\
\hline
\end{tabular}

Table 1: Simulation Example Parameters 


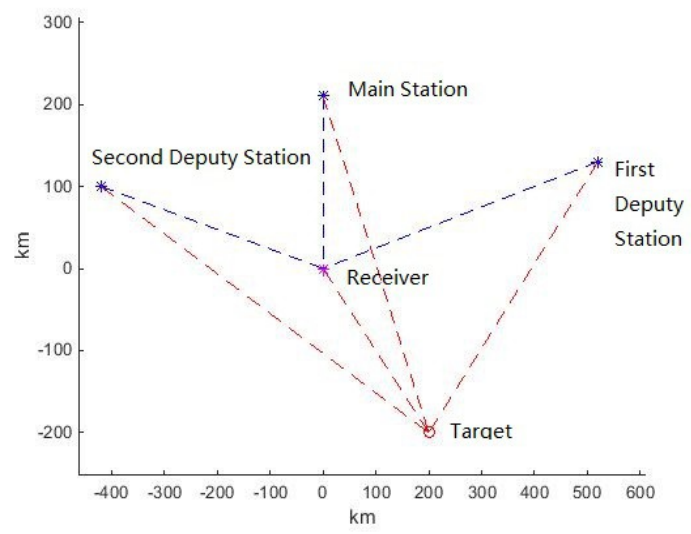

Figure4: Geometrical Configuration of the Simulation Environment
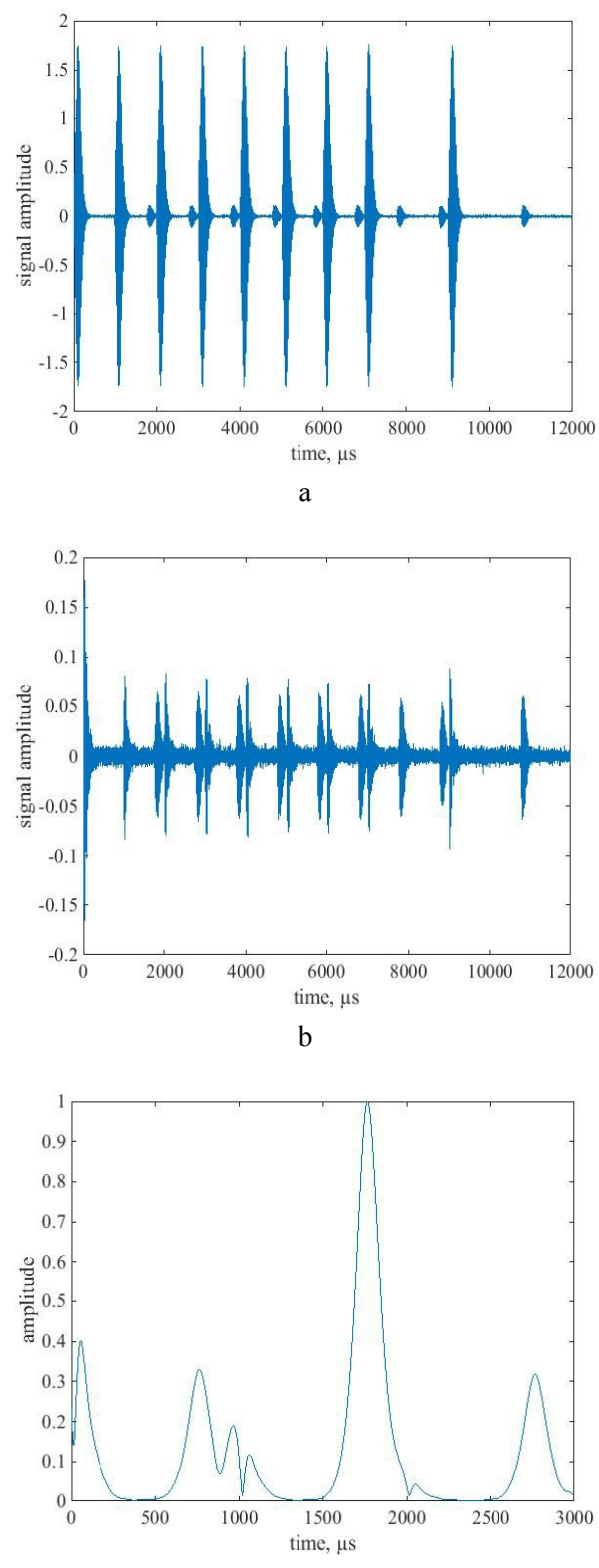

Figure 5: Processing Result of Simulation Signal

a Simulated data

b Interference suppression result

c Correlation result. 


\section{Conclusion}

In this paper, a passive detection method based on Loran- $\mathrm{C}$ is proposed and the advantages of Loran-C signal using for the detection of sea targets are elaborated. Based on the characteristics of Loran-C signal, this paper describes the processing schemes and associated techniques in detail. According to the processing results illustrated in Section 5, there is no doubt that the proposed method has the capability to detect sea target beyond long range. Although there are many advantages of Loran-C signal for the detection of sea target, it must be pointed out that Doppler information is barely utilized because of the low carrier frequency of Loran-C signal. There is still more work for continuation and improvements when the method is applied for real conditions.

\section{References}

[1] P. E . Howland, D. Maksimiuk, G. Reitsma. FM radio based bistatic radar [J]. IEE ProceedingsRadar, Sonar and Navigation, 2005, 152(3): 107-115

[2] H. D. Griffiths, C. J. Baker. Passive coherent location radar systems. Part 1: performance prediction $[\mathrm{C}] / /$ Radar, Sonar and Navigation, IEE Proceedings-. IET, 2005, 152(3): 153-159

[3] C. J. Baker, H. D. Griffiths, I. Papoutsis. Passive coherent location radar systems. Part 2: Waveform properties[J]. IEE Proceedings-Radar, Sonar and Navigation, 2005, 152(3): 160-168

[4] D. K. P. Tan, H. Sun, Y. Lu. Passive radar using global system for mobile communication signal: theory, implementation and measurements[C]//Radar, Sonar and Navigation, IEE Proceedings-. IET, 2005, 152(3): 116-123

[5] D. K. P. Tan, M. Lesturgie, H. Sun. Signal analysis of airborne passive radar using transmissions of opportunity[C], 2011 IEEE CIE International Conference on. IEEE, 2011, 1: 169-172

[6] A. Mohammed, D. Last. IFFT technique for skywave detection in Loran-C receivers[J]. Electronics Letters, 2001, 37(6): 398-400

[7] J. A. Pierce. An introduction to Loran[J]. Proceedings of the IRE, 1946, 34(5): 216-234

[8] S. S. Haykin. Adaptive filter theory[M]. Pearson Education India, 2008: 535-600 